\title{
Outcomes of Hand-Assisted Laparoscopic Surgery for Colorectal Disease in an Emergency Setting
}

\author{
Yoon Suk Lee, M.D., Ph.D. \\ Department of Surgery, Seoul St. Mary's Hospital, School of Medicine, The Catholic University of Korea, Seoul, Korea
}

Corresponding author

Yoon Suk Lee

Department of Surgery, Seoul St. Mary's Hospital, School of Medicine, The Catholic University of Korea, 222 Banpo-daero, Seocho-gu, Seoul 06591, Korea

Tel: +82-2-2258-6095, Fax: +82-2-595-2282, E-mail: yslee@ catholic.ac.kr

Since first introduction of laparoscopic surgery in the field of colorectal disease, ${ }^{1}$ laparoscopic surgery has replaced the open surgery in almost every procedure in colorectal disease. Even in colorectal cancer, better short-term outcomes and acceptable oncological outcomes had been established by controlled randomized trials, and considered as an alternative to open surgery for colorectal cancer. ${ }^{2-4}$ Because of the technical difficulties and steep learning curve, hand-assisted laparoscopic surgery (HALS) was introduced as the bridge to the laparoscopic surgery in the early period of laparoscopic colorectal surgery. However the role of HALS was diminished along with increased popularity of laparoscopic surgery.

Emergency setting of colorectal disease including obstruction and perforation results in distended, vulnerable, and edematous tissue; it is very challenging to perform laparoscopic surgery. As the authors said in this article, traction or pushing of tissue with carefully adjusted pressure, blunt dissection between inflamed tissues with minimal injury, and digital vascular control, all of which allow complex laparoscopic operations to be performed safely and effectively in emergency situation. So using HALS can be a good surgical option in colorectal emergency, but morbidities and mortalities can be increased in colorectal emergency and several studies reported high morbidities and mortalities in colorectal emer- gency regardless of the technique used. ${ }^{5,6}$ So during HALS for colorectal emergency, surgeons should not hesitate to convert to open surgery depending on the patients conditions. Conversion to open surgery does not mean treatment failure but mean changing treatment plan.

Laparoscopic surgery in colorectal emergency had better short-term outcomes compared to open surgery. HALS is the one of the approach methods of minimally invasive surgeries; it also has better short-term benefit when compared to conventional open surgery. But there few studies which compare the HALS and open surgery. If the authors conduct next comparative study of the short-term clinical outcomes between HALS and open surgery in colorectal emergency, it will be a very informative study.

\section{REFERENCES}

1) Jacobs M, Verdeja JC, Goldstein HS. Minimally Invasive Colon Resection (Laparoscopic Colectomy). Surg Laparosc Endosc 1991;1:144-150.

2) Fleshman J, Sargent DJ, Green E, et al. Laparoscopic colectomy for cancer is not inferior to open surgery based on 5-year data from the COST Study Group trial. Ann Surg 2007;246:655-662; discussion 662-654. 
3) Jeong S-Y, Park JW, Nam BH, et al. Open versus laparoscopic surgery for mid-rectal or low-rectal cancer after neoadjuvant chemoradiotherapy (COREAN trial): survival outcomes of an open-label, non-inferiority, randomised controlled trial. Lancet Oncol 2014;15:767-774.

4) Guillou PJ, Quirke P, Thorpe H, et al. Short-term endpoints of conventional versus laparoscopic-assisted surgery in patients with colorectal cancer (MRC CLASICC trial): multicentre, randomised controlled trial. Lancet 2005;365:1718-1726.
5) Biondo S, Kreisler E, Millan M, et al. Impact of surgical specialization on emergency colorectal surgery outcomes. Arch Surg 2010;145:79-86.

6) Skala K, Gervaz P, Buchs N, et al. Risk factors for mortality-morbidity after emergency-urgent colorectal surgery. Int J Colorectal Dis 2009;24:311-316.

7) Kim IY, Kim BR, Kim YW. Outcomes of laparoscopic and open surgery for colorectal cancer in the emergency setting. In Vivo 2015;29:295-300. 